International Journal of Aeronautical Science \& Aerospace Research (IJASAR)

ISSN: $2470-4415$

\title{
Necessary Modifications to the Strap-Down Inertial Navigation System Model for Non-Earth-Based Users
}

Tamer Mekky Ahmed

Research Article

Head of Spacecraft Dynamics and Control Department, Space Division, National Authority for Remote Sensing and Space Sciences, Cairo, Egypt.

\begin{abstract}
Strap-down inertial navigation system (SDINS) is considered as a highly sophisticated dead reckoning system. The SDINS consists mainly of two triads the first is the three gyros triad, and the second is the three accelerometers triad. Modeling the SDINS has achieved a lot of importance in the literature, especially for surveying applications. However, using the SDINS for spacecraft applications imply some modifications to the SDINS deterministic models used for surveying applications. Traditional models of the SDINS include the effect of the earth rotational rate on the SDINS instruments (gyros, and accelerometers). This effect must be removed from the instrument model if the SDINS is to be used onboard of a spacecraft simply because the spacecraft doesn't rest on the earth, and thus doesn't sense the earth's rotational rate. Necessary modifications of the accompanying algorithms are derived analytically in the research at hand.
\end{abstract}

Keywords: Strap-Down; INS; Accelerometer; Gyro.

\section{*Corresponding Author:}

Tamer Mekky Ahmed

Head of spacecraft dynamics and control department, Space Division,

National Authority for Remote Sensing and Space Sciences, 23 Joseph

Broz Teto Street, El Nozha El Gedida, Behind Cairo Airport, Cairo,

Egypt.

Tel: 00201117154301

E-mail: tamermekky@hotmail.com

Received: November 10, 2014

Accepted: November 17, 2014

Published: December 18, 2014

Citation: Tamer Mekky Ahmed (2014) Necessary Modifications to the Strap-Down Inertial Navigation System Model for Non-Earth-Based Users. Int J Aeronautics Aerospace Res. 1(2), 11-20. doi: http://dx.doi. org/10.19070/2470-4415-140002

Copyright: Tamer Mekky Ahmed ${ }^{\circ} 2014$ This is an open-access article distributed under the terms of the Creative Commons Attribution License, which permits unrestricted use, distribution and reproduction in any medium, provided the original author and source are credited.

\section{Acceleration Measurement}

Different researches and text books had discussed the inertial navigation system, or some of its components such as [1-3] and [4]. The inertial navigation system accelerometer consists of a proof mass $m$ suspended from a case by a pair of springs, as illustrated in Figure. 1. Referring to Figure. 1, the input axis is indicated by the arrow. If the body to which the system is attached is accelerating along this axis by an acceleration, a, the proof mass will be displaced from its equilibrium position by an amount, $\mathrm{x}$. From Newton's law, the force affecting on the proof mass is equal to the mass times the acceleration. And from Hook's law, the force generated by the springs are equal to the equivalent spring constant, $\mathrm{K}$, times the displacement $\mathrm{x}$. Thus, from Newton's law we find that

$F=m a=K x$

Therefore the measured acceleration is

$$
a=\frac{K}{m} x
$$

Consequently, acceleration in the plus direction causes the proof mass to move downward indicating positive acceleration. The displacement $\mathrm{x}$ is sensed using a pick-off and scaled to provide the value of acceleration. The proof mass equilibrium position is calibrated for zero acceleration. If the accelerometer is set on a bench in the earth's environment, the gravity force will shift the proof mass downward w.r.t the case indicating positive acceleration. However, the gravitational acceleration is downward. For this reason, equation(1) becomes

$F=m(a-g)=K x$

And the measured acceleration is

$\mathrm{a}-\mathrm{g}=\frac{\mathrm{K}}{\mathrm{m}} x=f$

Where, $f$ is the specific force.

\section{Commonly used Reference Frames}

The earth centered earth fixed (ECEF) coordinates are defined 
Figure 1. Accelerometer configuration.

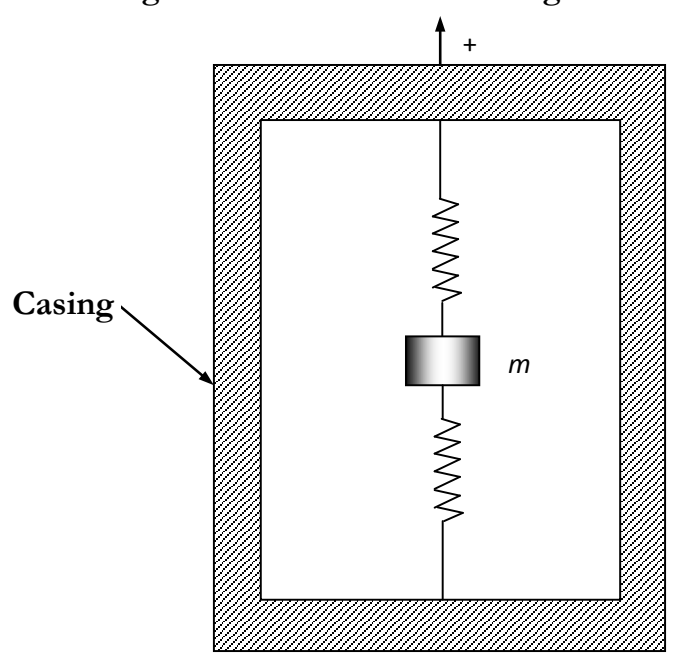

such that the $X_{E}$ axis points towards the intersection between the Greenwich meridian and the earth's equatorial plane, this point has a longitude and latitude $(0,0)$ deg respectively [5]. The $Z_{E}$ axis of the ECEF axis is the earth's rotational axis about itself. The $Y_{E}$ axis is chosen to complete the right hand rule system.

The local-level (navigation) frame is defined such that $X$ axis $(E)$ points toward the east, $Y$ axis $(N)$ points to the north, and the $Z$ axis (UP) points in the direction from the earth center to the INS origin. The ECEF and the navigation frame are illustrated in Figure 2. The Geocentric-Inertial system has its origin at the earth's center. The fundamental plane is the equatorial plane. The positive $Z$ axis points in the direction of the North Pole, the positive $X$ axis points in the direction of the vernal equinox on the first day of spring, and the positive $Y$ axis completes the right handed set of coordinates. It is important to keep in mind that the inertial coordinate system is non rotating w.r.t the stars (except for precession of the equinoxes).

The angle between the vernal equinox unit vector and Greenwich meridian is called $\alpha_{g}$-the "Greenwich sidereal time." What we need is a convenient way to calculate the angle $\alpha_{g}$ for any date and time of day. If we knew what $\alpha_{g}$ was on a particular day and time we could calculate $\alpha_{g}$ for any future time since we know that in one day the earth turns through 1.0027379093 complete rotations on its axis.

Suppose we take the value of $\alpha_{g}$ at $0^{\mathrm{h}}$ UT on 1 January of a particular year and call it $\alpha_{g 0}$. If, in addition, we express time in decimal fractions of a day, we can convert a particular day and time into a single number of days which have been elapsed since our "time zero." If we call this number $D$, then

$\alpha_{g}=\alpha_{g_{0}}+1.0027379093 \times 360^{\circ} \times D$ [In Degrees]

or

$\alpha_{g}=\alpha_{g o}+1.0027379093 \times 2 \pi \times D$ [In Radians]

Where

$\alpha_{g}=1.74933340 \mathrm{rad}$ at $1 / 1 / 19700^{\mathrm{h}}: 0^{\mathrm{m}}: 0^{\mathrm{s}}$.

The transformation matrix from inertial to ECEF coordinates is now computed from

$$
\left[\begin{array}{l}
X_{E} \\
Y_{E} \\
Z_{E}
\end{array}\right]=\left[\begin{array}{ccc}
\cos \alpha_{g} & \sin \alpha_{g} & 0 \\
-\sin \alpha_{g} & \cos \alpha_{g} & 0 \\
0 & 0 & 1
\end{array}\right]\left[\begin{array}{c}
X_{I} \\
Y_{I} \\
Z_{I}
\end{array}\right]
$$

The body system of axes is defined in Figure. 4.

\section{The oretical Measurements of a Moving Gyro Triad}

The actual measurements of the accelerometers are the sum of the nominal measurements and the errors. If the accelerometers are stationary and miss-leveled by $r, p$, and $\theta$ errors about the x, y, and $z$ body axes, they will measure a part of the gravitational force according to the relation

$$
\mathrm{F}=A_{r p}\left[\begin{array}{c}
0 \\
0 \\
-g
\end{array}\right]
$$

Where, $A_{r \phi \theta}$ is the miss-alignment matrix. The nominal terrestrial usage of the gyros involves sensing the earth rotational components. As illustrated in Figure. 5, it is evident that the relation between the east velocity component and the longitude could be expressed as

$$
\stackrel{o}{\lambda}=\frac{V_{E}}{R_{\phi}}=\frac{V_{E}}{(R+h) \cos \phi}
$$

Referring to Figure. 6, we could also write

$$
\stackrel{o}{\phi}=\frac{V_{N}}{R+h}
$$

Where $\mathrm{h}$ is the spacecraft height. Finally, the local-level frame angular velocity components are

$$
\omega_{E}=-\varphi=\frac{V_{N}}{R+h}
$$


Figure. 2 ECEF and navigation frames.

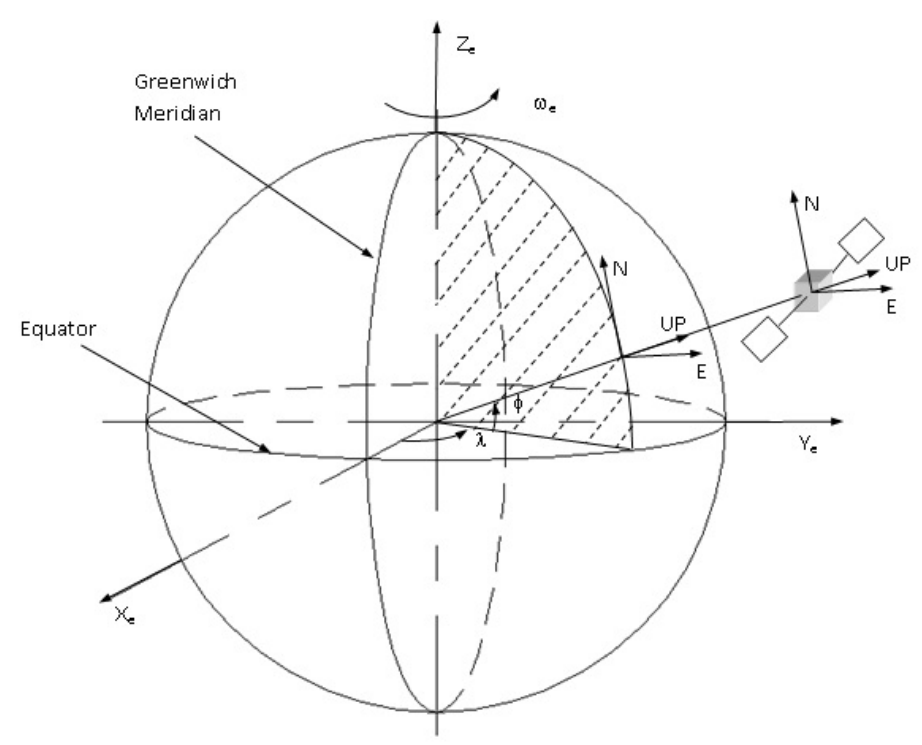

Figure 3. ECEF and Geocentric-Inertial axes

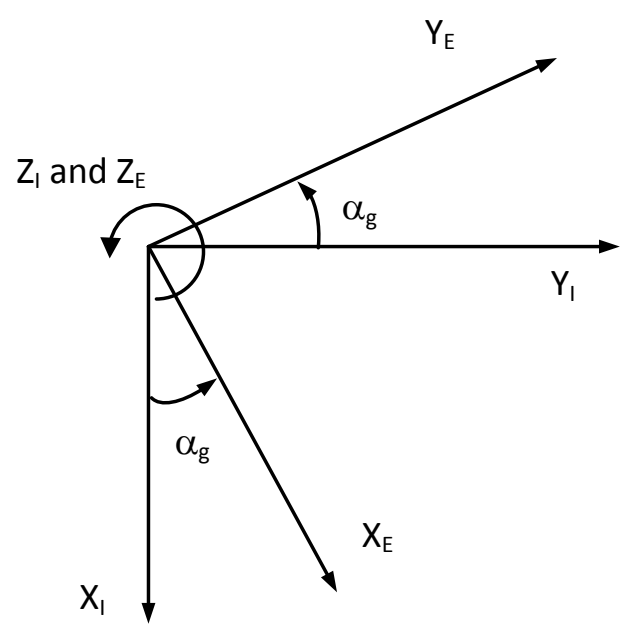

Figure 4. Spacecraft Body axes.

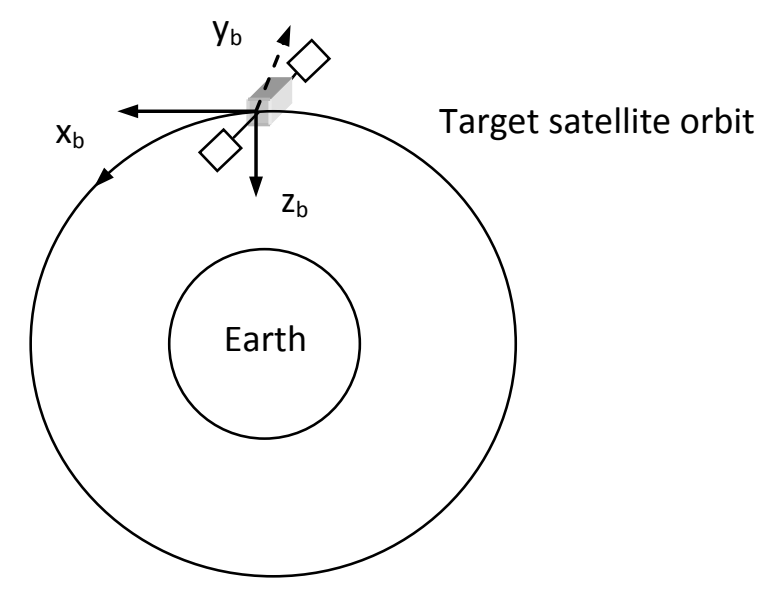


Figure 5. Relation between the east velocity component and the longitude

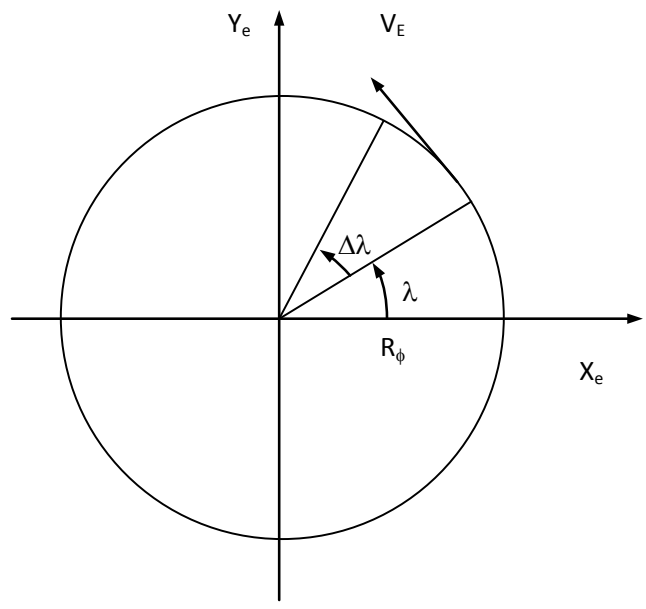

Figure 6. Relation between the east velocity component and the latitude

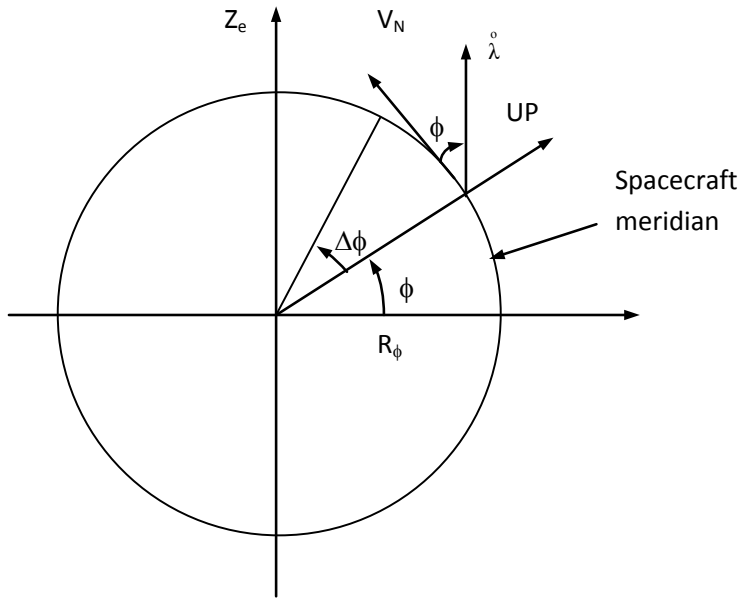

\section{Modeling the INS}

$\omega_{N}=\stackrel{\circ}{\lambda} \cos \varphi+\omega_{\mathrm{e}} \cos \varphi=\frac{V_{E}}{R+h}+\omega_{\mathrm{e}} \cos \varphi$

$\omega_{\mathrm{UP}}=\lambda \sin \varphi+\omega_{\mathrm{e}} \sin \varphi+=\tan \phi+\omega_{\mathrm{e}} \sin \phi$

In this treatment we have assumed that the body frame and the inertial frame are aligned together. The relationship between the navigation frame and the INS body frame is usually established through either a stationary alignment process, or by continuous external velocity information from GPS receiver. Thus, the attitude angles are used in generating the rotation between the bframe and the navigation frame. The rotation rates measured by the gyros are used to constantly update the transformation matrix between the body frame and the navigation frame. This transformation is used to transform the measured acceleration from the body frame to the navigation frame (in the case of strap down INS). Once transformed, integrating the acceleration twice will provide the INS position difference w.r.t. an initial point. However, as mentioned before, accelerometer measurements include both the platform and gravity, and hence knowledge of the gravitational field is mandatory to determine the platform acceleration. It is obvious that the INS is fundamentally dependent on an accurate knowledge of the initial position, velocity, and attitude of the moving platform prior to the start of INS operation.

\section{Mathematical Notations}

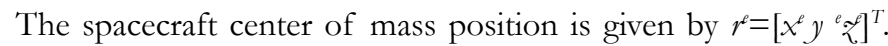
Transformation matrices are used to transform any navigation vector from one computational frame to another. As an example, the position vector in the e-frame (earth fixed frame), $r^{e}$, is transformed to the i-frame (inertial frame) to give ri through the

transformation matrix $R_{e}^{i}$, and hence

$$
r^{i}=R_{e}^{i} r^{e}
$$

Transformation from the i-frame to the e-frame is established throughout the inverse transformation, Therefore,

$$
r^{e}=\left(R_{e}^{i}\right)^{-1} r^{i}=R_{i}^{e} r^{i}
$$

And the angular velocity of the e frame w.r.t. the $i$ frame is denoted as $\omega_{i e}$. If this angular velocity is expressed in the e-frame, it will be denoted $\omega_{i e}^{e}$. The rotation between two coordinate frames can be expressed as a sum of rotations between different coordinate frames as

$$
\omega_{i e}^{e}=\omega_{i l}^{e}+\omega_{i l}^{e}
$$


Rotation can be represented in two ways, an angular velocity vector, and a skew symmetric matrix comprising the same vector components. For example, the angular velocity vector may be represented either in a vector form $\omega_{i e}^{e}=\left[\omega_{\mathrm{x}} \omega_{\mathrm{y}} \omega_{\mathrm{z}}\right]^{\mathrm{T}}$, or in the skew matrix form

$\Omega_{i e}^{e}=\left[\begin{array}{ccc}0 & -\omega_{z} & \omega_{y} \\ \omega_{z} & 0 & -\omega_{x} \\ -\omega_{y} & \omega_{x} & 0\end{array}\right]$

Similarly, the velocity vector could be expressed as

$\mathrm{v}^{\mathrm{e}}=\left[\begin{array}{c}V_{x} \\ V_{y} \\ V_{z}\end{array}\right] \& \mathrm{~V}^{\mathrm{e}}=\left[\begin{array}{ccc}0 & -V_{z} & V_{y} \\ V_{z} & 0 & -V_{x} \\ -V_{y} & V_{x} & 0\end{array}\right]$

It is also important to note that coordinate transformations apply also to angular velocity vectors. As an example, the angular velocity vector, $\omega_{i e}^{e}$, could be transformed to the inertial frame using the relation

$$
\omega_{i e}^{i}=R_{e}^{i} \omega_{i e}^{e}
$$

The equivalent transformation between two skew-symmetric matrices has the special form

$$
\Omega_{i e}^{i}=R_{e}^{i} \Omega_{i e}^{e} R_{i}^{e}
$$

If we have two vectors $a$ and $b$, their cross product $a \mathrm{~b}$ can be expressed in terms of their skew symmetric matrices (A or B) as

$$
a \times b=A b=-B a
$$

\section{Time Derivative of the Rotation Matrix}

For a fixed point in a certain coordinate frame (body-frame) the position vector ( $r$ ) can be transformed from the body frame, $b$, to the inertial frame, i, by the transformation

$$
r^{i}=R_{b}^{i} r^{b}
$$

Differentiation of both sides of equation (23) assuming that $r^{b}$ is constant (i.e. the point in spacecraft body is assumed to have a pure rotational motion without any translation) will give

$$
\stackrel{o}{r^{i}}=\stackrel{o}{i} R_{b}^{b}
$$

The velocity of the body could be computed in the inertial frame from

$$
\mathrm{Vi}=r^{o}=\omega_{i b}^{i} \times r^{i}
$$

Using equation (22)we could write equation (25) in the form

$$
r^{i}=\Omega_{i b}^{i} r^{i}
$$

Substitution using equation (21), into equation (26) gives

$$
r^{i}=R_{b}^{i} \Omega_{i b}^{b} R_{i}^{b} r^{i}
$$

From equation (23) we could write equation (27) in the form

$$
r^{i}=R_{b}^{i} \Omega_{i b}^{b} R_{i}^{b} R_{b}^{i} r^{b}
$$

Since the matrix $R_{i}^{b}$ is the inverse of the $R_{b}^{i}$ matrix, then $R_{i}^{b}$ $R_{b}^{i}=\mathrm{I}$ and equation (28) is reduced to

$$
r^{i}=R_{b}^{i} \Omega_{i b}^{b} r^{b}
$$

Comparing equation (24) with equation (29) we could easily deduce that

$$
\stackrel{o}{R_{b}^{i}}=R_{b}^{i} \Omega_{i b}^{b}
$$

\section{Calculation of Position and Velocity Derivatives}

The vector $r^{a}$ is transformed from the a coordinate frame to the $\mathrm{b}$ coordinate frame according to the relation

$$
r^{b}=R^{b}+R_{a}^{b} r^{a}
$$

Where: $R^{b}$ is the vector directed from the $b$-frame origin to the $a$-frame origin, and $R_{a}^{b}$ is the matrix that transforms from the $\mathrm{a}$-frame to the b-frame. If the $a$-frame rotates w.r.t. the $b$-frame, then differentiation of equation (31) gives

$$
\frac{d}{d t} r^{b}=\frac{d}{d t} R^{b}+\frac{d}{d t}\left(R_{a}^{b} r^{a}\right)=\frac{d R_{b}}{d t}+\frac{d R_{a}^{b}}{d t} r^{a}+R_{a}^{b} \frac{d r^{a}}{d t}
$$

Substitution using equation (30), by replacing the subscript b with $a$ and the super script $i$ with $b$ into equation (32) gives

$\frac{d}{d t} r^{b}=\frac{d R_{b}}{d t}+R_{a}^{b} \Omega_{b a}^{a} r^{a}+R_{a}^{b} \frac{d r^{a}}{d t}=\frac{d R_{b}}{d t}+R_{a}^{b} \Omega_{b a}^{a} r^{a}+R_{a}^{b} \mathrm{~V}^{a}=\frac{d R_{b}}{d t}+R_{a}^{b}\left(\Omega_{b a}^{a} r^{a}+\mathrm{v}^{a}\right)$

Equation (33) has the form of the coriolis theorem which states that, If two coinciding frames of reference experience relative

angular rotation $\Omega_{b a}^{a}(t)$ with $r^{b}(\mathrm{t})=R_{a}^{b} r^{a}(t)$, and $R_{a}^{b}=R_{a}^{b}$

$\Omega_{b a}^{b}(t)$ then the time rate of change of the two coordinate systems are related by

$$
r^{b}(t)=R_{a}^{b}(t)\left[\Omega_{b a}^{a}(t) r^{a}(t)+r^{a}(t)\right]
$$




$$
\frac{d^{2} r^{b}}{d t^{2}}=\frac{d^{2} R_{b}}{d t^{2}}+\left[\frac{d}{d t}\left(R_{a}^{b}\right)\right]\left[\Omega_{b a}^{a} r^{a}+\mathrm{v}^{a}\right]+R_{a}^{b}\left[\Omega_{b a}^{a} \mathrm{~V}^{a}+\Omega_{b a}^{a} r^{a}+\frac{d^{2} r^{a}}{d t^{2}}\right]
$$

Substitution using equation (30) into equation (35) results in

$$
\frac{d^{2} r^{b}}{d t^{2}}=\frac{d^{2} R_{b}}{d t^{2}}+R_{a}^{b} \Omega_{b a}^{a}\left[\Omega_{b a}^{a} r^{a}+\mathrm{V}^{a}\right]+R_{a}^{b}\left[\Omega_{b a}^{a} \mathrm{~V}^{a}+\stackrel{\rho^{a}}{\Omega_{b a}} r^{a}+\frac{d^{2} r^{a}}{d t^{2}}\right]
$$

From which we could write

$$
\frac{d^{2} r^{b}}{d t^{2}}=\frac{d^{2} R_{b}}{d t^{2}}+R_{a}^{b}\left[2 \Omega_{b a}^{a} \mathrm{~V}^{a}+\Omega_{b a}^{a} \Omega_{b a}^{a} r^{a}+\stackrel{o}{a}_{b a}^{a} r^{a}+\frac{d^{2} r^{a}}{d t^{2}}\right]
$$

Mechanization of inertial frame: The inertial body velocity can be expressed using coriolis equation using the following relation

$\frac{d}{d t} r_{i}=\frac{d}{d t} r_{e}+\omega_{i e} \times r$

Differentiating equation (37) and letting $\mathrm{d} / \mathrm{dt} r_{e}=v_{e}$, we get

$$
\frac{d^{2}}{d t^{2}} r_{i}=\frac{d}{d t} \mathbf{V}_{e i}+\frac{d}{d t}\left[\omega_{i e} \times r\right]_{i}
$$

Application of equation (37) to the second term in the right hand side of equation (38) gives

$$
\begin{aligned}
& \frac{d}{d t}\left[\omega_{i e} \times r\right]_{i}=\frac{d}{d t}\left[\omega_{i e} \times r\right]_{e}+\omega_{i e} \times\left[\omega_{i e} \times r\right]=\omega_{i e} \times \frac{d}{d t} r \\
& +\left[\frac{d}{d t} \omega_{i e}\right] \times r+\omega_{i e} \times\left[\omega_{i e} \times r\right]
\end{aligned}
$$

But the earth tures rate is constant, therefore $\left[\frac{d}{d t}\right]=0$, and equation (39) becom

$$
\frac{d}{d t}\left[\omega_{i e} \times r\right]_{i}=\omega_{i e} \times \mathrm{V}_{e}+\omega_{i e} \times\left[\omega_{i e} \times r\right]
$$

Equation (40) could now be substituted instead of the second term of equation (38) and this substitution results in

$\frac{d^{2}}{d t^{2}} r_{i}=\frac{d}{d t} \mathrm{~V}_{e i}+\omega_{i e} \times \mathrm{V}_{e}+\omega_{i e} \times\left[\omega_{i e} \times r\right]$

From which it is evident that

$$
\frac{d}{d t} \mathbf{V}_{e i}=\frac{d^{2}}{d t^{2}} r_{i}-\omega_{i e} \times \mathrm{V}_{e}-\omega_{i e} \times\left[\omega_{i e} \times r\right]
$$

Equation (4) says that $a=f+g$, where a $=\frac{d^{2}}{d t^{2}} r_{i}$. Consequently,
equation (42) becomes $\frac{d}{d t} \mathbf{V}_{e i}=f-\omega_{i e} \times \mathrm{V}_{e}-\omega_{i e} \times\left[\omega_{i e} \times r\right]+g$

Looking carefully into equation (43) we could easily see that:

1- $f:$ Is the specific force acceleration to which the INS is subjected.

2- $\omega_{i e} \times \mathrm{V}_{e}$ : Is the coriolis acceleration.

3- $\omega_{i e} \times\left[\omega_{i e} \times r\right]:$ Is the centripetal acceleration caused by the earth rotation with respect to an inertial frame.

4- $-\omega_{i e} \times\left[\omega_{i e} \times r\right]+$ g: is the centrifugal acceleration representing the local gravity vector, gl. So equation (43) could be written as

$\frac{d}{d t} \mathbf{V}_{e_{i}}=f-\omega_{i e} \times \mathbf{v}_{e}+\mathrm{g}_{l}$

This equation could be expressed in the inertial frame of reference as

$\mathrm{V}_{e}^{i}=f^{\prime}-\omega_{i e}^{i} \times \mathrm{V}_{e}^{i}+g_{l}^{i}$

The accelerometer outputs are the specific forces measured in body axes rather than inertial system of axes, if a strap down package is used. Thus, equation (45) should be written as

$\stackrel{o}{i}_{e}^{i}=R_{b}^{i} f^{b_{-}} \omega_{i e}^{i} \times \mathrm{V}_{e}^{i}+g_{l}^{i}$

Mechanization of earth centered fixed frame: Using Coriolis equation, the ground velocity expressed in ECEF coordinate sys-

tem, $\mathrm{V}_{e}^{e}$, is differentiated with respect to time to give

$\frac{d}{d t} \mathbf{V}_{e e}=\frac{d}{d t} \mathbf{V}_{e_{i}}-\omega_{i e} \times \mathbf{V}_{e}$

Substitution from equation (45) results in

$\frac{d}{d t} \mathbf{V}_{e e}=f-\omega_{i e} \times \mathbf{V}_{e}+\mathrm{g}_{l}-\omega_{i e} \times \mathbf{V}_{e}$

$\frac{d}{d t} \mathrm{~V}_{e_{e}}=f-2 \quad \omega_{i e} \times \mathrm{V}_{e}+\mathrm{g}_{l}$

Expressing equation (48) in the ECEF axes gives

$\mathrm{V}_{e}^{e}=\mathrm{f}^{\mathrm{e}}-2 \quad \omega_{i e}^{e} \times \mathrm{V}_{e}^{e}+g_{l}^{e}$

Mechanization of navigation frame: Mechanization of the navigation (local level) frame proceeds as the earth centered fixed frame mechanization. The derivatives of the ground velocity in the (local level) frame and the inertial frame of references are related by 
$\frac{d}{d t} \mathbf{V}_{e_{i}}=\frac{d}{d t} \mathbf{V}_{e_{i}}-\omega_{i l} \times \mathrm{V}_{e}=\frac{d}{d t} \mathbf{V}_{e_{i}-}\left(\omega_{i e}+\omega_{e l}\right) \times \mathrm{V}_{e}$

Substitution from equation (45) into equation (50) gives

$\frac{d}{d t} \mathbf{V}_{e i}=\mathrm{f}^{\prime}-\omega_{i e} \times \mathrm{V}_{e}+\left(\omega_{i e}+\omega_{e l}\right) \times \mathrm{V}_{e}=\mathrm{f}-\left(2 \omega_{i e}+\omega_{e l}\right) \times \mathrm{V}_{e}+\mathrm{g}$ l

Equation (51) is expressed in the local level frame by

$\mathrm{V}_{e}^{l}=\mathrm{f}^{l}-\left(2 \omega_{i e}^{l}+\omega_{e l}^{l}\right) \times{\underset{o}{o}}_{\mathbf{V}_{e}^{l}}^{l}+g_{l}^{l}$

\section{Mechanization equations for land based users}

Referring to Figure.6, The earth rotation angular velocity vector is in the same direction of $\lambda$, Thus for earth based users equations (12), (13), and (14) include the components of the earth's angular velocity vector. As a result, let's define

$\omega_{\dot{k}}^{l}=\left[\begin{array}{c}0 \\ \omega_{e} \cos \ddot{\mathrm{o}} \\ \omega_{e} \sin \ddot{\mathrm{o}}\end{array}\right], \omega_{k}^{l}=\left[\begin{array}{c}\frac{-\mathrm{V}_{\mathrm{N}}}{\mathrm{R}+\mathrm{h}} \\ \frac{\mathrm{V}_{\mathrm{E}}}{\mathrm{R}+\mathrm{h}} \\ \frac{\mathrm{V}_{\mathrm{E}}}{\mathrm{R}+\mathrm{h}} \tan \ddot{\mathrm{o}}\end{array}\right]$, and $\quad \omega_{t}^{l}=\left[\begin{array}{c}\grave{\mathrm{u}}_{\mathrm{E}} \\ \grave{\mathrm{u}}_{\mathrm{N}} \\ \grave{\mathrm{u}} \mathrm{\textrm {V }}\end{array}\right]$

So equations (12), (13), and (14) could be put in the form

$\omega_{i l}^{l}=\omega_{i e}^{l}+\omega_{e l}^{l}$

The first important note is related to the attitude mechanization equation. Applying equation (30) in the local level navigation frame results in:

$\stackrel{o}{R_{b}^{l}}=R_{b}^{l} \Omega_{l b}^{b}$

But we know that

$\Omega_{l b}^{b}=\Omega_{l i}^{b}+\Omega_{i b}^{b}=-\Omega_{i l}^{b}+\Omega_{i b}^{b}=\Omega_{i b}^{b}-\Omega_{i l}^{b}$

And

$\Omega_{i l}^{b}=\Omega_{i e}^{b}+\Omega_{e l}^{b}$

Substitution from equation (57) into equation (56) gives

$\Omega_{l b}^{b}=\Omega_{i b}^{b}\left(\Omega_{i e}^{b}+\Omega_{e l}^{b}\right)$

Consequently the attitude mechanization equations become

$$
\stackrel{o}{R_{b}^{l}}=R_{b}^{l}\left(\Omega_{i b}^{b}-\Omega_{i e}^{b}-\Omega_{e l}^{b}\right)
$$

Where the $\Omega_{i b}^{b}$ matrix is obtained from gyro measurements,

$\Omega_{e l}^{b}$ is the skew symmetric matrix corresponding to the vector

$\omega_{e l}^{b}$ which could be obtained from

$$
\omega_{k}^{b}=R_{l}^{b} \omega_{k}^{l}=R_{l}^{b}\left[\begin{array}{c}
\frac{-\mathrm{V}_{\mathrm{N}}}{\mathrm{R}+\mathrm{h}} \\
\frac{\mathrm{V}_{\mathrm{E}}}{\mathrm{R}+\mathrm{h}} \\
\frac{\mathrm{V}_{\mathrm{E}} \tan \ddot{\mathrm{o}}}{\mathrm{R}+\mathrm{h}}
\end{array}\right]
$$

And similarly we could compute the skew symmetric matrix $\Omega_{i e}^{b}$ from the corresponding angular velocity vector $\omega_{i e}^{b}$ which could be computed from

$\omega_{i e}^{b}=R_{l}^{b}\left[\begin{array}{c}0 \\ \grave{\mathrm{u}}_{\mathrm{e}} \cos \phi \\ \grave{\mathrm{u}}_{\mathrm{e}} \sin \phi\end{array}\right]$

And $R_{b}^{l}$ is the transformation matrix from the local level to the body frame defined by

$R_{b}^{l}=\left[\begin{array}{ccc}\cos A-\sin A \sin P \sin r & -\sin A \cos P & \cos A \sin R+\sin A \sin P \cos R \\ \sin A \cos r+\cos A \sin P \sin r & \cos A \cos P & \sin A \sin r-\cos A \sin P \cos r \\ -\cos P \sin r & \sin P & \cos P \cos r\end{array}\right]$

With $\mathrm{P}$, the pitch angle (inclination), $\mathrm{r}$ is the roll angle, $A$ is the azimuth angle.

Finally, the transformation matrix from the local level to the earth fixed frame is given by

$$
R_{l}^{e}=\left[\begin{array}{ccc}
-\sin \ddot{\mathrm{e}} & -\operatorname{sinö~} \operatorname{cosë} & \operatorname{cosö~cosë~} \\
\cos \ddot{\mathrm{e}} & -\operatorname{sinö~sin} \ddot{\mathrm{e}} & \cos \mathrm{sin} \\
0 & \cos 0 \ddot{ } & \operatorname{sinö}
\end{array}\right]
$$

Quaternion attitude representation: Quaternion parameters are introduced to describe the rotation of the b-frame w.r.t. the local level frame. The transformation matrix that transforms a vector from the reference frame to the body frame is a proper real orthogonal one. This means that it preserves the lengths of vectors and the angles between them, and also the inverse of the matrix is simply its transpose. So if $\mathrm{A}$ is the transformation matrix that transforms a vector from the reference to the body coordinate system, its transpose $\mathrm{A}^{\mathrm{T}}$ will transform that vector back to the reference system.

It is also known that a proper real orthogonal 33 matrix has at least one eigenvector with eigenvalue unity [7] and hence there exists a unit vector, $\hat{e}$ that isn't changed by A so that

$A \hat{e}=\hat{e}$

Which means that the vector $\hat{e}$ has the same components along both body and reference axes, so $\hat{e}$ is the axis of rotation. This demonstrates Euler's theorem: "the most general displacement of a rigid body with one point fixed is a rotation about some axis".

Referring to the transformation matrices described earlier, for a 
single rotation about single axis, we find that: for a rotation with single angle $\phi$ the trace (sum of all diagonal elements) of the corresponding transformation matrix is

$\operatorname{tr}(A(\phi))=1+2 \cos \phi$

Generalizing this rule for any sequence of rotations, we may transform any successive rotations to a single rotation by an angle $\phi$ about a unit vector (axis of rotation) such that

$$
\cos \Phi=\frac{1}{2}[t(A)-1]
$$

Examining this equation, we find that it has two solutions for the Euler axis / angle $\phi$, which differ in sign only. The two solutions have the rotation axis vectors in opposite directions.

This means that a rotation about the vector $\hat{e}$ by an angle $\phi$ is equivalent to a rotation about the vector $-\hat{e}$ by $-\phi$

The Euler symmetric parameters are defined in terms of Euler axis / angle and the components of the axis of rotation $\hat{e}$ as follows

$$
\begin{aligned}
& q 1=e 1 \sin (\phi / 2) \\
& q^{2}=e 2 \sin (\phi / 2) \\
& q^{3}=e 3 \sin (\phi / 2) \\
& q 4=\cos (\phi / 2)
\end{aligned}
$$

The four Euler symmetric parameters satisfy also the constraint

$$
q_{1}^{2}+q_{2}^{2}+q_{3}^{2}+q_{4}^{2}=1
$$

The four parameters could be regarded as the components of a quaternion vector,

$$
q=\left[\begin{array}{l}
q_{1} \\
q_{2} \\
q_{3} \\
q_{4}
\end{array}\right]=\left[\begin{array}{l}
\mathrm{q} \\
q_{4}
\end{array}\right]
$$

The direction cosine matrix could be expressed in terms of quaternion as in equation (70).

$$
A(q)=\left[\begin{array}{ccc}
q_{1}^{2}-q_{2}^{2}-q_{3}^{2}+q_{4}^{2} & 2\left(q_{1} q_{2}-q_{3} q_{4}\right) & 2\left(q_{1} q_{3}+q_{2} q_{4}\right) \\
2\left(q_{1} q_{2}+q_{3} q_{4}\right) & -q_{1}^{2}+q_{2}^{2}-q_{3}^{2}+q_{4}^{2} & 2\left(q_{3} q_{2}-q_{1} q_{4}\right) \\
2\left(q_{1} q_{3}-q_{2} q_{4}\right) & 2\left(q_{3} q_{2}+q_{1} q_{4}\right) & -q_{1}^{2}-q_{2}^{2}+q_{3}^{2}+q_{4}^{2}
\end{array}\right]
$$

And also we could inversely express Euler symmetric parameters in terms of the direction cosine matrix as follows

$$
\begin{aligned}
& q_{1}= \pm 0.5\left(1+A_{11}-A_{22}-A_{33}\right)^{\frac{1}{2}} \\
& q_{2}= \pm 0.5\left(1-A_{11}+A_{22}-A_{33}\right)^{\frac{1}{2}} \\
& q_{3}= \pm 0.5\left(1-A_{11}-A_{22}+A_{33}\right)^{\frac{1}{2}}
\end{aligned}
$$

$q_{4}= \pm 0.5\left(1+A_{11}+A_{22}+A_{33}\right)^{\frac{1}{2}}$

Attitude Mechanization Equations in terms of Quaternion

Based on the pre-discussed principals we could write

$$
\omega_{i b}^{b}=\omega_{i l}^{b}+\omega_{l b}^{b}
$$

Where $\omega_{i b}^{b}$ is sensed by the gyroscopes. Thus,

$$
\omega_{l b}^{b}=\omega_{i b}^{b}-\omega_{i l}^{b}
$$

And similarly,

$$
\omega_{i l}^{b}=\omega_{i e}^{b}+\omega_{e l}^{b}
$$

But we know that, $\omega_{i e}^{b}=R_{l}^{b} \omega_{i e}^{l}$ and $\omega_{e l}^{b}=R_{l}^{b} \omega_{e l}^{l}$. Thus we could write equation (74) as

$\omega_{i l}^{b}=R_{l}^{b}\left(\omega_{i e}^{l}+\omega_{e l}^{l}\right)=R_{l}^{b}\left(\left[\begin{array}{c}\frac{-\mathrm{V}_{\mathrm{N}}}{\mathrm{R}+\mathrm{h}} \\ \frac{\mathrm{V}_{\mathrm{E}}}{\mathrm{R}+\mathrm{h}} \\ \frac{\mathrm{V}_{\mathrm{E}}}{\mathrm{R}+\mathrm{h}} \tan \ddot{\mathrm{o}}\end{array}\right]+\left[\begin{array}{c}0 \\ \grave{\mathrm{u}}_{\mathrm{e}} \cos \phi \\ \grave{\mathrm{u}}_{\mathrm{e}} \sin \phi\end{array}\right]\right)$

Substitution from equation (75) into equation (73) gives

$$
\omega_{l b}^{b}=\omega_{i b}^{b}-R_{l}^{b}\left(\left[\begin{array}{c}
\frac{-\mathrm{V}_{\mathrm{N}}}{\mathrm{R}+\mathrm{h}} \\
\frac{\mathrm{V}_{\mathrm{E}}}{\mathrm{R}+\mathrm{h}} \\
\frac{\mathrm{V}_{\mathrm{E}} \tan \ddot{\mathrm{o}}}{\mathrm{R}+\mathrm{h}}
\end{array}\right]+\left[\begin{array}{c}
0 \\
\mathrm{ù}_{\mathrm{e}} \cos \phi \\
\grave{\mathrm{u}}_{\mathrm{e}} \sin \phi
\end{array}\right]\right)
$$

In order to combine the parameters for two individual rotations we find that

$\left[A\left(q^{\prime \prime}\right)\right]=\left[A\left(q^{\prime}\right)\right][A(q)]$

The resulting quaternion $q^{\prime \prime}$ could be found from the final attitude matrix, or it could be in another different way [7]. If $q^{\prime \prime}$ represents the orientation of the body frame with respect to the reference frame at time $t+\Delta t, q$ represents it at time then $q^{\prime}$ represents the orientation of the body frame relative to the position that it occupied at time $t$, thus we could write

$$
q_{1}^{\prime}=e_{u} \sin \frac{\Delta \phi}{2}
$$

$$
q_{2}^{\prime}=e_{v} \sin \frac{\Delta \phi}{2}
$$




$$
\begin{gathered}
q_{3}^{\prime}=e_{w} \sin \frac{\Delta \phi}{2} \\
q_{4}^{\prime}=\cos \frac{\Delta \phi}{2}
\end{gathered}
$$

Where eu, ev and ew are the components of the rotation axis unit vector (i.e. they are in the direction of $\mathrm{p}, \mathrm{q}$ and $\mathrm{r}$ ), $\Delta \phi=\omega \Delta t=$ $\sqrt{p^{2}+q^{2}+r^{2}} \quad \Delta t$ (this is valid only for an infinitesimal time

interval $\Delta \mathrm{t}$ ), also we may write

$$
q^{\prime \prime}=q q^{\prime}
$$

Or in a matrix form

$$
q^{\prime \prime}=\left[\begin{array}{cccc}
q_{4}^{\prime} & q_{3}^{\prime} & -q_{2}^{\prime} & q_{1}^{\prime} \\
-q_{3}^{\prime} & q_{4}^{\prime} & q_{1}^{\prime} & q_{2}^{\prime} \\
q_{2}^{\prime} & -q_{1}^{\prime} & q_{4}^{\prime} & q_{3}^{\prime} \\
-q_{1}^{\prime} & -q_{2}^{\prime} & -q_{3}^{\prime} & q_{4}^{\prime}
\end{array}\right] q
$$

Replacing $q^{\prime}$ by the change in Euler axis / angle

$$
q^{\prime \prime}=\left[\begin{array}{cccc}
\cos \frac{\Delta \phi}{2} & e_{w} \sin \frac{\Delta \phi}{2} & -e_{v} \sin \frac{\Delta \phi}{2} & e_{u} \sin \frac{\Delta \phi}{2} \\
-e_{w} \sin \frac{\Delta \phi}{2} & \cos \frac{\Delta \phi}{2} & e_{u} \sin \frac{\Delta \phi}{2} & e_{v} \sin \frac{\Delta \phi}{2} \\
e_{v} \sin \frac{\Delta \phi}{2} & -e_{u} \sin \frac{\Delta \phi}{2} & \cos \frac{\Delta \phi}{2} & e_{w} \sin \frac{\Delta \phi}{2} \\
-e_{u} \sin \frac{\Delta \phi}{2} & -e_{v} \sin \frac{\Delta \phi}{2} & -e_{w} \sin \frac{\Delta \phi}{2} & \cos \frac{\Delta \phi}{2}
\end{array}\right] q
$$

Thus we may possibly write

$$
q(t+\Delta t)=\left\{\cos \frac{\Delta \phi}{2} I+\sin \frac{\Delta \phi}{2}\left[\begin{array}{cccc}
0 & e_{w} & -e_{v} & e_{u} \\
-e_{w} & 0 & e_{u} & e_{v} \\
e_{v} & -e_{u} & 0 & e_{w} \\
-e_{u} & -e_{v} & -e_{w} & 0
\end{array}\right]\right\} q(t)
$$

Where I is the fourth order identity matrix. In addition, all the right hand side terms are evaluated at time $t$. The former equation is mostly useful if the axis of rotation doesn't change over the time interval $\Delta t$. For the case of attitude dynamics and prediction, equation (81) may be converted to a differential equation, though it may be used as it is to reduce the integration effort. Also small angles approximation will be used with the assumption of infinitesimal time period $\Delta t$ to get

$$
\cos \frac{\Delta \Phi}{2} \approx 1 \text { and } \sin \frac{\Delta \Phi}{2} \approx \frac{\Delta \Phi}{2} \approx \frac{\omega \Delta t}{2}
$$

Noting that $\omega=\omega \hat{e}=\left[\omega_{X}, \omega_{Y}, \omega_{Z}\right]^{T}$ is the instantaneous angular velocity vector Finally we could write

$q(t+\Delta t) \approx[I D+\Omega(\Delta t / 2)] q(t)$

And $\Omega$ is the skew-symmetric matrix
$\Omega=\left[\begin{array}{cccc}0 & \omega_{z} & -\omega_{y} & \omega_{x} \\ -\omega_{z} & 0 & \omega_{x} & \omega_{y} \\ \omega_{y} & -\omega_{x} & 0 & \omega_{z} \\ -\omega_{x} & -\omega_{y} & -\omega_{z} & 0\end{array}\right]$

Where $\omega_{x}, \omega_{y}$, and $\omega_{z}$ are the components of the angular velocity vector $\omega_{l b}^{b}$.

To go over the main points, the INS mechanization equations for land based users are summarized as

\section{Position mechanization equation}

The position of a moving platform described in the l-frame is expressed in terms of the curvilinear coordinates $(\phi, \lambda, h)$, where $\phi$ is the latitude, $b$ is the longitude, $h$ is the altitude. Thus, we could write

$$
r=\left[\begin{array}{l}
\phi \\
\lambda \\
h
\end{array}\right]
$$

and from equations (10) and (11) we could write

$$
\stackrel{o}{r}^{l}=\left[\begin{array}{c}
o \\
\phi \\
o \\
\lambda \\
h \\
h
\end{array}\right]=\left[\begin{array}{ccc}
0 & \frac{1}{R+h} & 0 \\
\frac{1}{(R+h) \cos \phi} & 0 & 0 \\
0 & 0 & 1
\end{array}\right]\left[\begin{array}{c}
V_{E} \\
V_{N} \\
V_{U}
\end{array}\right]=D^{-1} \quad \mathbf{v}_{e}^{l}
$$

\section{Velocity mechanization equation}

Using equation (52), we could write

$$
\stackrel{o^{l}}{\mathrm{v}_{e}}=\mathrm{f}^{\prime}-\left(2 \omega_{i e}^{l}+\omega_{e l}^{l}\right) \times \mathrm{V}_{e}^{l}+g_{l}^{l}
$$

Thus,

$$
\stackrel{o}{l}^{l}=R_{b}^{l} \rho^{p}-\left(2 \omega_{i e}^{l}+\omega_{e l}^{l}\right) \times \mathbf{V}_{e}^{l}+g_{l}^{l}
$$

Equation (86) implies the existence of a gravity model. To simplify the problem, we present the simplest possible gravity model defined by [6]

$g(h)=g(0) /(1+h / R) 2$

Where, $g(0)=9.780318 \mathrm{~m} / \mathrm{s}^{2}$, and $g(h)$ is the magnitude of earth gravitational vector defined in the local level frame by $g=\left[\begin{array}{ll}0 & 0\end{array}\right.$ $-\mathrm{g}(b)]^{\mathrm{T}}$. The local gravity vector is thus

$$
g_{l}^{l}=\mathrm{g} \quad-\omega_{i e} \times\left[\omega_{i e} \times r\right]
$$




\section{Attitude mechanization equations}

The mechanization equations of attitude are given in equation

(59) as $\stackrel{o}{R}_{b}^{l}=R_{b}^{l}\left(\Omega_{i b}^{b}-\Omega_{i e}^{b}-\Omega_{e l}^{b}\right)$ or alternatively in equation (82) as $\mathrm{q}(t+\Delta t) \approx[\mathrm{ID}+\Omega(\Delta t / 2)] \mathrm{q}(t)$, and $\Omega_{l b}^{b}$ is given by its corresponding form of $\omega_{l b}^{b}$ given by equation (76).

\section{Modification of the Mechanization Equations for Space based users}

Looking carefully into equations (52), (53), and (54) we could deduce some important notes. First, the term $\omega_{i e}^{l}$, represents a component of the earth's rotation sensed by a stationary INS (i.e. $\mathrm{V}_{\mathrm{E}}=\mathrm{V}_{\mathrm{N}}=\mathrm{V}_{\mathrm{UP}}=0 \mathrm{~m} / \mathrm{sec}$ ) on the earth's surface. For space based users, this term won't be sensed by the INS at all. So, $\omega_{i e}^{l}=0$, and $\omega_{i l}^{l}=\omega_{e l}^{l}$. The second important note is related to the attitude mechanization equation. Applying equation (30) in the local level navigation frame results in:

$\stackrel{o}{R_{b}^{l}}=R_{b}^{l} \Omega_{l b}^{b}$

But we know that

$\Omega_{l b}^{b}=\Omega_{l i}^{b}+\Omega_{i b}^{b}=-\Omega_{i l}^{b}+\Omega_{i b}^{b}=\Omega_{i b}^{b}-\Omega_{i l}^{b}$

And

$\Omega_{i l}^{b}=\Omega_{i e}^{b}+\Omega_{e l}^{b}$

Substitution from equation (91) into equation (90) gives

$\Omega_{l b}^{b}=\Omega_{i b}^{b}-\left(\Omega_{i e}^{b}+\Omega_{e l}^{b}\right)$

But $\Omega_{i e}^{b}$ is the skew-symmetric matrix corresponding to $\omega_{i e}^{b}$ which is equal to $R_{l}^{b} \omega_{i e}^{l}$, and we also know that $\omega_{i e}^{l}=0$. Thus, equation (92) reduces to

$$
\Omega_{l b}^{b}=\Omega_{i b}^{b}-\Omega_{e l}^{b}
$$

Consequently the attitude mechanization equations become

$$
\stackrel{o}{R_{b}^{l}}=R_{b}^{l}\left(\Omega_{i b}^{b}-\Omega_{e l}^{b}\right)
$$

Where the $\Omega_{i b}^{b}$ matrix is obtained from gyro measurements, $\Omega_{e l}^{b}$ is the skew symmetric matrix corresponding to the vector $\omega_{e l}^{b}$ which could be obtained from

$$
\omega_{e l}^{b}=R_{l}^{b} \omega_{e l}^{l}=R_{l}^{b}\left[\begin{array}{c}
\frac{-\mathrm{V}_{\mathrm{N}}}{\mathrm{R}+\mathrm{h}} \\
\frac{\mathrm{V}_{\mathrm{E}}}{\mathrm{R}+\mathrm{h}} \\
\frac{\mathrm{V}_{\mathrm{E}}}{\mathrm{R}+\mathrm{h}} \tan \ddot{\mathrm{o}}
\end{array}\right]
$$

\section{Velocity mechanization equation}

Using equation (52) in addition to that, $\omega_{i e}^{l}=0$, we could write

$$
\mathrm{V}_{e}{ }^{l}=\mathrm{f}^{l}\left(2 \omega_{i e}^{l}+\omega_{e l}^{l}\right) \times \mathrm{V}_{e}^{l}+g_{l}^{l}=\mathrm{f}^{l}-\omega_{e l}^{l} \times \mathrm{V}_{e}^{l}+g_{l}^{l}
$$

Thus,

$$
{\stackrel{o}{\mathrm{~V}_{e}}}^{l}=R_{b}^{l} \mathrm{f}^{b}-\omega_{e l}^{l} \times \mathrm{V}_{e}^{l}+g_{l}^{l}
$$

The gravity model given by equation (88) should now be modified to reflect the absence of earth rotation sensing by the accelerometers (recalling that $\omega_{i e}^{l}=0$ ). So the gravity model becomes

$$
g_{l}^{l}=\mathrm{g}-\omega_{i e} \times\left[\omega_{i e} \times r\right]=\mathbf{g}
$$

\section{Attitude mechanization equations}

The mechanization equations of attitude are given in equation (94) as ${\stackrel{o}{R_{b}^{l}}}^{l}=R_{b}^{l}\left(\Omega_{i b}^{b}-\Omega_{e l}^{b}\right)$ or alternatively in equation (1.99) as $\mathrm{q}(\mathrm{t}+\Delta \mathrm{t}) \approx\left[\mathrm{ID}+\Omega_{l b}^{b}(\Delta \mathrm{t} / 2)\right] \mathrm{q}(\mathrm{t})$, and $\Omega_{l b}^{b}$ is now given by equation (93) instead of its corresponding form of $\omega_{l b}^{b}$ given by equation (76).

\section{Conclusion}

The most important conclusion coming out from this research is that the application through which the SDINS is used affects the associated mechanization equations and the gravity model. For terrestrial applications, the SDINS model is developed clearly with the appropriate systems of axes. If the SDINS is utilized by a user who isn't resting on the earth the SDINS model must be modified according to section (7).

\section{References}

[1]. Habib, T., New AlgorithmS of Nonlinear Spacecraft Attitude Control via Attitude, Angular Velocity, and Orbit Estimation Based on the Earth's Magnetic Field”, PhD thesis, Aerospace Department, Cairo University, 2009.

[2]. Sumit, J., et al," Preliminary Design of Solar Powered Unmanned Aerial Vehicle", Applied Mechanics and Materials, Vol.225, Issue 1, 2012, pp. 315322.

[3]. Jashnani, S., et al," Sizing and preliminary hardware testing of solar powered UAV", The Egyptian Journal of Remote Sensing and Space Sciences, Vol.16, 2013, pp. 189-198.

[4]. Liu, X., et al," A Method for SINS Alignment with Large Initial Misalignment Angles Based on Kalman Filter with Parameters Resetting", Mathematical Problems in Engineering, Vol.2014, dx.doi.org/10.1155/2014/346291.

[5]. Habib, T.," The Global Positioning System Application to Satellite Position and Attitude determination", MSc thesis, Aerospace Department, Cairo University, 2003.

[6]. Shearman, and Bradsell, P.," Strap-down Inertial Navigation Technology", Peter Peregrinus Ltd., 1997.

[7]. Wertz, J. R., "Spacecraft Attitude Determination and Control", D. Reidel Publishing Company, 1997. 